Conclusions High admissions rates from OPD reflect the challenges of serving a geographically large and rural population. In the rainy season, dengue fever flourishes and fear of missing this diagnosis in febrile children may influence clinician behaviour during this period. Admission rates were highest in July with a second peak in September. OPD attendances peaked later (October). We suggest that road flooding in August and September restricts travel, limiting total attendances. With the state hospital's increasing role as a receiving centre from smaller hospitals, further study is needed to characterise the burden of disease in children presenting to hospital and guide service development in Myanmar.

\section{G458(P) CODING OF ADMISSION DIAGNOSIS IN A DISTRICT GENERAL HOSPITAL IN MYANMAR}

${ }^{1} \mathrm{E}$ Ivey, ${ }^{2} \mathrm{HH}$ Win, ${ }^{2} \mathrm{HMM}$ Chan, ${ }^{1} \mathrm{M}$ Wootton, ${ }^{3}$ I Maconochie. ${ }^{1}$ Global Child Health, Royal College of Paediatrics and Child Health, London, UK; ${ }^{2}$ Child Ward, Hpa An General Hospital, Hpa An, Myanmar; ${ }^{3}$ Paediatric Emergency Medicine, Imperial College Healthcare Trust NHS, London, UK

\subsection{6/archdischild-2020-rcpch.394}

Background With increasing numbers of children presenting to state and district general hospitals, including those transferred from smaller township and station hospitals, there is increasing need to understand the burden of disease presenting to hospital. The WHO recommends all countries use ICD-10 coding for reporting health data. There is little research to date describing the terms used to record admission diagnoses in paediatrics in Myanmar.

Aim To review the library of terms currently used to record admission diagnosis in a typical paediatric ward and map these against the ICD-10; to assess feasibility of applying this standardised system of coding across paediatrics in Myanmar.

Methods Logbook records from the Child Ward (CW) were reviewed for the period May 2018 - April 2019. All primary admission diagnoses were recorded and grouped by body system. Without editing or inferring meanings of the terms, these were individually tested for matching against ICD-10 codes.

Results Over a 12 month period, 343 different admission diagnoses were recorded. It was possible to match $90.9 \%$ diagnoses to ICD-10 codes. However, many could only be matched to 'not specified' codes and $18.5 \%$ could only be matched to terms in Chapter XVIII (symptoms and signs not elsewhere classified). Some chapters had no matches across the 1 year review period.

Conclusions We have shown that it is possible to use ICD-10 but the application of such a complex system has multiple challenges in a resource-limited setting. The heavy use of 'not specified' codes reflects a lack of diagnostic precision, which is multifactorial, influenced by factors including limited availability of investigations, variable access to specialist advice and lack of training in coding. A coding system for this setting would need to factor in the inherent diagnostic uncertainty and be user-friendly enough to require little clinician training, whilst yielding clinically and academically robust data.

We propose that a standardised approach to diagnosis recording, with modifications to overcome the challenges of directly applying ICD-10 in a low-resource setting, would be useful here and in other global healthcare facilities to guide resource allocation and to monitor changes in patterns of illness according to interventions.

\section{G459(P) VACS: VACCINATION AWARENESS IN CHILDREN STUDY - A STUDY TO LOOK INTO PERCEPTIONS AND ASSUMPTIONS AMONG PARENTS AND CLINICIANS}

C Devanahalli, S Kapoor, D Burnside, R Radcliffe. University Hospital Leicester NHS Trust, Leicester, UK

\subsection{6/archdischild-2020-rcpch.395}

Introduction The Childhood Vaccination Coverage Statistics of England, 2018-19, shows a drop in vaccination rate of primary vaccination ranging from 0.2 to 1.0 percentage points. Of note the average uptake of annual flu vaccination across UK has been 50\% amongst children. Despite having a robust vaccination programme there is declining trend in vaccination uptake in United Kingdom for the past few years. We were keen to explore the uptake rate of childhood vaccination in our population; to evaluate this further we explored knowledge and perceptions amongst parents and clinicians of the 'up-to-date' vaccination status in our cohort.

Methods The study was conducted in a busy tertiary paediatric hospital. We included children more than 3 months of age admitted to the paediatric wards over one month period.

Study was conducted in two phases:

First phase: Consisted of face to face questionnaire (Interview) conducted with parents. We then retrospectively looked at the vaccination status in the clerking notes. As the final step we then checked the accuracy of this information with the red book.

Second phase: Knowledge assessment for junior doctors in our paediatric department performed by survey. The doctors involved in this study were excluded from this assessment.

Results Data from 50 children was analysed which showed $100 \%$ uptake of routine childhood vaccinations. Flu vaccination uptake was $50 \%$. Vaccination status was documented in $50 \%$ of the initial clerking notes. Red book was not available to cross check the accuracy in most children. There were significant gaps in knowledge among junior doctors from their assessment.

Conclusion Junior doctors can act as important messengers in promoting vaccination awareness. Our study highlights that the perception of being fully vaccinated by parents and clinicians may not always be accurate. Parents do not always carry red books and there is a need for alternate means of information sharing amongst health professions.

Address gaps in knowledge among junior doctors will empower them to play an important role in health promotion of vaccine preventable disease.

\section{G460(P) TEACHING PAEDIATRIC RESUSCITATION IN A RESOURCE LIMITED SETTING IN WEST AFRICA}

${ }^{1}$ ON Oketah, ${ }^{2} \mathrm{R}$ Macdonell, ${ }^{3} \mathrm{~S}$ Koe, ${ }^{4} \mathrm{I}$ Okafor. ${ }^{1}$ Paediatrics and Adolescent Medicine, University College London Hospital, London, UK; ${ }^{2}$ Paediatrics, Royal College of Physicians of Ireland, Dublin, Ireland; '3Paediatrics, Children's Health Ireland at Tallaght, Dublin, Ireland; ${ }^{4}$ Paediatric Emergency Medicine, Children's Health Ireland at Temple Street, Dublin, Ireland

10.1136/archdischild-2020-rcpch.396 